\title{
Article
}

\section{Eye Infections Caused by Filamentous Fungi: Spectrum and Antifungal Susceptibility of the Prevailing Agents in Germany}

\author{
Grit Walther ${ }^{1, *}$, Anna Zimmermann ${ }^{2}{ }^{\oplus}$, Johanna Theuersbacher ${ }^{3}$, Kerstin Kaerger ${ }^{1}$, Marie von Lilienfeld-Toal ${ }^{1,4}$, \\ Mathias Roth ${ }^{5} \mathbb{( D}$, Daniel Kampik ${ }^{3}$, Gerd Geerling ${ }^{5}\left(\mathbb{D}\right.$ and Oliver Kurzai ${ }^{1,2} \mathbb{E}$
}

1 National Reference Center for Invasive Fungal Infections (NRZMyk), Leibniz Institute for Natural Product Research and Infection Biology—Hans Knöll Institute, Adolf-Reichwein-Str. 23, 07745 Jena, Germany; kkaerger@web.de (K.K.); Marie.von_Lilienfeld-Toal@med.uni-jena.de (M.v.L.-T.); okurzai@hygiene.uni-wuerzburg.de (O.K.)

2 Institute for Hygiene and Microbiology, University of Würzburg, 97080 Würzburg, Germany; anna.zimmermann@uni-wuerzburg.de

3 Department of Ophthalmology, University Hospital Würzburg, 97080 Würzburg, Germany; j.theuersbacher@icloud.com (J.T.); Kampik_D@ukw.de (D.K.)

4 Department of Haematology and Medical Oncology, University Hospital Jena, 07747 Jena, Germany

5 Department of Ophthalmology, Heinrich-Heine University Düsseldorf, 40225 Düsseldorf, Germany; mathiasroth@gmx.net (M.R.); geerling@med.uni-duesseldorf.de (G.G.)

* Correspondence: Grit.Walther@leibniz-hki.de; Tel.: +49-3641-532-1038; Fax: +49-3641-939-6502

\section{check for}

updates

Citation: Walther, G.; Zimmermann, A.; Theuersbacher, J.; Kaerger, K.; von Lilienfeld-Toal, M.; Roth, M.; Kampik, D.; Geerling, G.; Kurzai, O. Eye Infections Caused by Filamentous Fungi: Spectrum and Antifungal Susceptibility of the Prevailing Agents in Germany. J. Fungi 2021, 7, 511. https://doi.org/10.3390/ jof7070511

Academic Editor: David S. Perlin

Received: 30 April 2021

Accepted: 17 June 2021

Published: 26 June 2021

Publisher's Note: MDPI stays neutral with regard to jurisdictional claims in published maps and institutional affiliations.

Copyright: (c) 2021 by the authors. Licensee MDPI, Basel, Switzerland. This article is an open access article distributed under the terms and conditions of the Creative Commons Attribution (CC BY) license (https:// creativecommons.org/licenses/by/ $4.0 /)$.

\begin{abstract}
Fungal eye infections can lead to loss of vision and blindness. The disease is most prevalent in the tropics, although case numbers in moderate climates are increasing as well. This study aimed to determine the dominating filamentous fungi causing eye infections in Germany and their antifungal susceptibility profiles in order to improve treatment, including cases with unidentified pathogenic fungi. As such, we studied all filamentous fungi isolated from the eye or associated materials that were sent to the NRZMyk between 2014 and 2020. All strains were molecularly identified and antifungal susceptibility testing according to the EUCAST protocol was performed for common species. In total, 242 strains of 66 species were received. Fusarium was the dominating genus, followed by Aspergillus, Purpureocillium, Alternaria, and Scedosporium. The most prevalent species in eye samples were Fusarium petroliphilum, F. keratoplasticum, and F. solani of the Fusarium solani species complex. The spectrum of species comprises less susceptible taxa for amphotericin B, natamycin, and azoles, including voriconazole. Natamycin is effective for most species but not for Aspergillus flavus or Purpureocillium spp. Some strains of F. solani show MICs higher than $16 \mathrm{mg} / \mathrm{L}$. Our data underline the importance of species identification for correct treatment.
\end{abstract}

Keywords: eye infection; fungal infection; keratitis; antifungal susceptibility; natamycin; Fusarium; Purpureocillium; Aspergillus; Alternaria; Scedosporium

\section{Introduction}

Eye infections caused by fungi are serious diseases that can lead to loss of vision and blindness. Sites of exogenous infections mainly include the cornea (keratitis), but also the vitreous body (endophthalmitis), often as consequence of progressing keratitis. According to estimates, more than one million people are affected by fungal keratitis annually and $8-11 \%$ of the patients lose the eye [1]. The disease is most prevalent in tropical and subtropical countries [2,3], but an increasing number of cases has also been reported in countries with moderate climates [4-10]. Numerous fungal species are known to cause keratitis. Clinically, wearing of soft contact lenses and ocular trauma is mostly associated with keratitis caused by filamentous fungi, whereas keratitis caused by Candida spp. most frequently occurs in patients with chronic eye disease, topical steroid use, or surgical intervention. The spectrum of filamentous fungi causing keratitis differs depending on the climate and geographic region, but Aspergillus and Fusarium species are the predominant 
causative agents worldwide [11-13]. In several countries, including India, Nepal, and the USA (Florida), Curvularia species play an important role as the cause of keratitis [12]. Data from the German Fungal Keratitis Registry have shown that roughly one-third of cases in Germany are caused by Candida species, one-third is Fusarium keratitis, and one-third is caused by other filamentous fungi, including Aspergillus spp. [14].

In the two most frequently isolated genera of filamentous fungi associated with eye infections, Fusarium and Aspergillus, numerous new species have been described in the last years based on DNA sequence data [15-19]. As a consequence, morphology based approaches often only allow the identification of species complexes or sections, respectively. Even molecular species identification by sequencing of the nuclear ribosomal internal transcribed spacer (ITS) region that is used as a universal marker in fungi [20] cannot discriminate all species in these genera [21,22]. Consequently, exact species identification is often lacking in published case series of keratitis caused by filamentous fungi.

The taxonomy and nomenclature of the genus Fusarium are currently controversial. Some authors favor the use of the name Fusarium and a wide generic concept including the FSSC [23], while other authors support a smaller generic concept that splits the genus Fusarium into several genera (e.g., Neocosmospora), primarily based on characteristics of their fruiting bodies (perithecia), but also on the asexual morphs [24]. Due to the fact that Fusarium is of great importance in medical mycology because it is one of the opportunistic fungal genera that is recognized morphologically, we use the first concept and the nomenclature suggested by Geiser et al. [23].

Currently, the treatment of fungal keratitis is mainly based on the polyene drugs natamycin (also known as pimaricin) or amphotericin B and the azole antifungal voriconazole. All three drugs can be applied topically, whereas evidence suggests that only voriconazole should be considered for systemic treatment [25]. Natamycin and amphotericin B largely have good in vitro activity against Fusarium spp. [26]. Voriconazole is clinically effective against Fusarium spp., despite variable in vitro activity. It is effective against some species of Aspergillus [27] and Scedosporium [28]. Local natamycin was associated with better visual acuity after infection and a reduced number of corneal perforations or the need to perform therapeutic keratoplasty compared to monotherapy by voriconazole [25].

To assess the epidemiological situation of keratitis caused by filamentous fungi in Germany and provide representative data on the in vitro antifungal susceptibility of the causative agents, this study aims to answer the following questions: (1) Which filamentous fungal species are causing keratitis in Germany? (2) What are the antifungal susceptibility profiles of these species? (3) Is natamycin effective against all fungal taxa causing eye infections? (4) Are there differences in the minimum inhibitory concentrations (MICs) of natamycin among Fusarium species?

To answer these questions, we studied all filamentous fungi from eye infections that were sent to the National Reference Center for Invasive Fungal Infections (NRZMyk) between January 2014 and December 2020. Molecular species identification was performed for all isolates using reliable markers established for the respective genera. Antifungal susceptibility was tested in vitro according to the EUCAST protocol for all Fusarium strains, as well as for other more frequent species.

On the basis of molecular species identifications, we showed that the prevalent agents of eye infections in Germany belong to the genera Fusarium, Aspergillus, Purpureocillium, Alternaria, and Scedosporium. The antifungal susceptibility profiles revealed that natamycin is effective for most species but not for Aspergillus flavus or the genus Purpureocillium, representing common agents of eye infections in Germany. Within the genus Fusarium, only some F. solani isolates showed MICs $>16 \mathrm{mg} / \mathrm{L}$.

\section{Material and Methods}

\subsection{Isolates}

Filamentous fungi isolated from the eye (corneal and conjunctival swabs and scrapings, aspirates of the anterior chamber or the vitreous body) or from eye-associated materials 
such as contact lenses or cleaning solutions that were sent to the NRZMyk between January 2014 and December 2020 were included in the study of the spectrum of species involved in eye infections. Due to research activities in this area, the NRZMyk receives a high number of samples from cases of filamentous keratitis. In case several isolates of the same species from the same patient were received, only the initial isolate was included. For antifungal susceptibility testing (AFST), additional strains from other sources were included for species represented by a small number of eye-related isolates in order to cover the variability of the respective species. Isolates related to ocular infections, as well as isolates from other sources that were used for AFST, were deposited in the Jena Microbial Resource Collection (JMRC). Strain numbers, GenBank accession numbers of the identifying sequences, sources, and the minimum inhibitory concentrations are given in Table S1.

\subsection{Molecular Species Identification}

Genomic DNA was extracted from 2- to 7-day-old cultures grown on 4\% malt extract agar (Difco), as described before [9]. Depending on the genus, different markers were amplified by PCR for species identification. Table S2 provides the primers used for PCR and sequencing of each genus, as well as their annealing temperatures. The SeqMan program v. 11.0.0. (DNASTAR, Lasergene, Madison, WI, USA) was used to construct consensus sequences. Species were identified by using the BLAST tool in GenBank (Available online: blast.ncbi.nlm.nih.gov /Blast.cgi?PAGE_TYPE=BlastSearch, accessed on 31 March 2021). Sequences were deposited at GenBank (Available online: www.ncbi.nlm.nih.gov/genbank/, accessed on 31 March 2021) and their accession numbers are listed in Table S1.

\subsection{Antifungal Susceptibility Testing}

In vitro antifungal susceptibilities of all Fusarium species and all other species that were isolated at least three times from infected eyes or eye-associated material were performed by broth microdilution technique following the European Committee on Antimicrobial Susceptibility Testing (EUCAST) standard methodology [29]. If the number of isolates from eyes or eye-associated material was below 10 for a species, additional antifungal susceptibility tests were performed with isolates of this species from other sources for a better coverage of the variability of the species (Table S1). The following antifungals were tested: amphotericin B (AMB; European Pharmacopoeia, Strasbourg, France); caspofungin (CAS; MSD, Rahway, NJ, USA), isavuconazol (ISA; Basilea Pharmaceutica International Ltd., Basel, Switzerland); itraconazole (ITZ), natamycin (NAT) (Chemicalpoint, Deisenhofen, Germany); posaconazole (PCZ; MSD, Rahway, NJ, USA); and voriconazole (VCZ; Pfizer Inc., Peapack, NJ, USA).

Fungi were grown on MEA for 2 to 7 days at $35{ }^{\circ} \mathrm{C}$ (Aspergillus), $30{ }^{\circ} \mathrm{C}$ (Alternaria, Fusarium, Scedosporium), or room temperature (Cladosporium). Spore suspensions were counted with a hemocytometer. MIC endpoints were defined as 100\% reduction in growth and were determined visually using a mirror after $48 \mathrm{~h}$ of incubation at $35^{\circ} \mathrm{C}$, except for Cladosporium strains, for which the endpoints were determined after $72 \mathrm{~h}$ at $30{ }^{\circ} \mathrm{C}$ due to their lower maximum temperature of growth. For caspofungin, minimum effective concentrations (MECs) were determined by reading the microplates with the aid of an inverted microscope. Aspergillus fumigatus ATCC 204305 and Candida parapsilosis ATCC 22019 served as reference strains. For the calculation of geometric means, high off-scale MICs/MECs were raised to the next higher concentration.

\section{Results}

\subsection{Spectrum of Filamentous Fungi Causing Eye Infections in Germany}

In the 7-year period from 2014 to 2020, the NRZMyk received 242 strains of 66 species related to eye infections of 234 patients. In six cases, we received two different species from the same patient, while in a single case we received three different species isolated from the same patient. Of the 66 species received in total, 35 species were exclusively isolated from 
the eye, 17 species were isolated from the eye and eye-associated material, and 14 species were isolated from eye-associated material only (Table 1 and Table S1).

Table 1. Spectrum of fungi isolated from infected eyes and associated materials (corneal and conjunctival swabs, aspirates of the anterior chamber and the vitreous body). For the FSSC, the numbering of the phylogenetic species is given in brackets.

\begin{tabular}{|c|c|c|}
\hline Species & No. of Isolates from Eye & No. of Isolates from Eye-Associated Materials \\
\hline Total strain number & $169(100 \%)$ & $73(100 \%)$ \\
\hline Alternaria & $8(4.7 \%)$ & $2(2.7 \%)$ \\
\hline Alternaria alternata & $8(4.7 \%)$ & 0 \\
\hline Alternaria hordeicola & 0 & $1(1.4 \%)$ \\
\hline Alternaria rosae & 0 & $1(1.4 \%)$ \\
\hline Arthrographis kalrae & $2(1.2 \%)$ & 0 \\
\hline Aspergillus & $22(13.0 \%)$ & $4(5.5 \%)$ \\
\hline Aspergillus cibarius & $1(0.6 \%)$ & 0 \\
\hline Aspergillus flavus & $6(3.6 \%)$ & $1(1.4 \%)$ \\
\hline Aspergillus fumigatus & $10(5.9 \%)$ & $1(1.4 \%)$ \\
\hline Aspergillus hiratsukae & $1(0.6 \%)$ & 0 \\
\hline Aspergillus sydowii & 0 & $1(1.4 \%)$ \\
\hline Aspergillus terreus & $1(0.6 \%)$ & 0 \\
\hline Aspergillus tubingensis & $2(1.2 \%)$ & $1(1.4 \%)$ \\
\hline Aspergillus udagawae & $1(0.6 \%)$ & 0 \\
\hline Aureobasidium pullulans & $1(0.6 \%)$ & 0 \\
\hline Chaetomium anastomosans & $1(0.6 \%)$ & 0 \\
\hline Cladosporium cladosporioides complex & $3(1.8 \%)$ & $1(1.4 \%)$ \\
\hline Coprinellus domesticus & $1(0.6 \%)$ & 0 \\
\hline Epicoccum mezzettii & $1(0.6 \%)$ & 0 \\
\hline Fusarium & $80(47.3 \%)$ & $55(75.3 \%)$ \\
\hline FDSC & $1(0.6 \%)$ & $4(5.5 \%)$ \\
\hline Fusarium dimerum & 0 & $4(5.5 \%)$ \\
\hline Fusarium sp. & $1(0.6 \%)$ & 0 \\
\hline FFSC & $12(7.1 \%)$ & $5(6.8 \%)$ \\
\hline Fusarium lactis & $1(0.6 \%)$ & 0 \\
\hline Fusarium musae & $2(1.2 \%)$ & $1(1.4 \%)$ \\
\hline Fusarium proliferatum & $7(4.1 \%)$ & $3(4.1 \%)$ \\
\hline Fusarium sacchari & $1(0.6 \%)$ & 0 \\
\hline Fusarium verticillioides & $1(0.6 \%)$ & $1(1.4 \%)$ \\
\hline FIESC & $1(0.6 \%)$ & 0 \\
\hline Fusarium equiseti & $1(0.6 \%)$ & 0 \\
\hline FOSC & $10(5.9 \%)$ & $33(45.2 \%)$ \\
\hline FRSC & 0 & $1(1.4 \%)$ \\
\hline Fusarium redolens & 0 & $1(1.4 \%)$ \\
\hline FSSC & $56(33.1 \%)$ & $12(16.4 \%)$ \\
\hline Fusarium bostrycoides (FSSC $25+35)$ & $3(1.8 \%)$ & 0 \\
\hline Fusarium cyanescens (FSSC 27) & $1(0.6 \%)$ & 0 \\
\hline Fusarium falciforme (FSSC $3+4)$ & $5(3.0 \%)$ & 0 \\
\hline Fusarium ferrugineum (FSSC 28) & 0 & $1(1.4 \%)$ \\
\hline Fusarium keratoplasticum (FSSC 2) & $13(7.7 \%)$ & $1(1.4 \%)$ \\
\hline Fusarium petroliphilum (FSSC 1) & $20(11.8 \%)$ & $7(9.6 \%)$ \\
\hline Fusarium metavorans (FSSC 6) & $2(1.2 \%)$ & 0 \\
\hline Fusarium pisi (FSSC 11) & 0 & $1(1.4 \%)$ \\
\hline Fusarium solani (FSSC 5) & $11(6.5 \%)$ & $3(4.1 \%)$ \\
\hline Fusarium stercicola (FSSC 44) & 0 & $1(1.4 \%)$ \\
\hline Fusarium tonkinense (FSSC 9) & $1(0.6 \%)$ & $1(1.4 \%)$ \\
\hline Gnomoniopsis idaeicola & 0 & $1(1.4 \%)$ \\
\hline Lecanicillium & $2(1.2 \%)$ & $2(2.7 \%)$ \\
\hline Lecanicillium attenuatum & 0 & $1(1.4 \%)$ \\
\hline Lecanicillium coprophilum & $2(1.2 \%)$ & $1(1.4 \%)$ \\
\hline
\end{tabular}


Table 1. Cont.

\begin{tabular}{|c|c|c|}
\hline Species & No. of Isolates from Eye & No. of Isolates from Eye-Associated Materials \\
\hline Lecythophora hoffmannii & $1(0.6 \%)$ & 0 \\
\hline Lichtheimia corymbifera & $1(0.6 \%)$ & 0 \\
\hline Lomentospora prolificans & $2(1.2 \%)$ & 0 \\
\hline Montagnula opulenta & 0 & $1(1.4 \%)$ \\
\hline Penicillium & $3(1.8 \%)$ & $2(2.7 \%)$ \\
\hline Penicillium chrysogenum & 0 & $1(1.4 \%)$ \\
\hline Penicillium citrinum & $1(0.6 \%)$ & 0 \\
\hline Penicillium crustosum & $1(0.6 \%)$ & 0 \\
\hline Penicillium rubens & $1(0.6 \%)$ & $1(1.4 \%)$ \\
\hline Peniophora lycii & $1(0.6 \%)$ & 0 \\
\hline Peroneutypa scoparia & $1(0.6 \%)$ & 0 \\
\hline Petriella setifera & $1(0.6 \%)$ & 0 \\
\hline Plectosphaerella & $1(0.6 \%)$ & $1(1.4 \%)$ \\
\hline Plectosphaerella cucumerina & $1(0.6 \%)$ & 0 \\
\hline Plectosphaerella sp. & 0 & $1(1.4 \%)$ \\
\hline Pseudopithomyces sp. & $1(0.6 \%)$ & 0 \\
\hline Purpureocillium & $21(12.4 \%)$ & 0 \\
\hline Purpureocillium lilacinum & $15(8.9 \%)$ & 0 \\
\hline Purpureocillium sodanum & $6(3.6 \%)$ & 0 \\
\hline Rhinocladiella similis & $1(0.6 \%)$ & 0 \\
\hline Sarocladium & $2(1.2 \%)$ & $2(2.7 \%)$ \\
\hline Sarocladium kiliense & $1(0.6 \%)$ & $1(1.4 \%)$ \\
\hline Sarocladium spinificis & $1(0.6 \%)$ & 0 \\
\hline Sarocladium strictum & 0 & $1(1.4 \%)$ \\
\hline Scedosporium & $8(4.7 \%)$ & $1(1.4 \%)$ \\
\hline Scedosporium apiospermum & $7(4.1 \%)$ & 0 \\
\hline Scedosporium dehoogii & $1(0.6 \%)$ & $1(1.4 \%)$ \\
\hline Schizophyllum commune & $1(0.6 \%)$ & 0 \\
\hline Scopulariopsis brevicaulis & $1(0.6 \%)$ & 0 \\
\hline Tintelnotia destructans & $2(1.2 \%)$ & $1(1.4 \%)$ \\
\hline
\end{tabular}

With few exceptions we were able to identify the strains molecularly by the markers listed in Table S2. One Fusarium strain (FDSC) could not be identified at the species level due to a lack of reference sequences in GenBank. For one strain of Plectosphaerella sp. and one strain of Pseudopithomyces sp., the ITS region was not discriminative enough to identify the species.

Among the isolates from the eye, Fusarium is the dominant genus (80 strains, $47.3 \%$ ), followed by Aspergillus (22 strains, 13.0\%), Purpureocillium (21 strains, 12.4\%), Alternaria (8 strains, 4.7\%), and Scedosporium (8 strains, 4.7\%) (Figure 1). Within the genus Fusarium, the Fusarium solani species complex (FSSC) is predominant, while the Fusarium fujikuroi species complex (FFSC) is the second most common and the Fusarium oxysporum species complex (FOSC) is the third most common (Table 1). With 8 species isolated from the eye, the FSSC is represented by the highest number of species. The most prevalent FSSC species are F. petroliphilum, F. keratoplasticum, and F. solani. Predominant species in the remaining genera are Purpureocillium lilacinum, Aspergillus fumigatus, A. flavus, Alternaria alternata, and Scedosporium apiospermum. Beside Purpureocillium lilacinum, the recently described sibling species Purpureocillium sodanum was identified in 6 cases. 

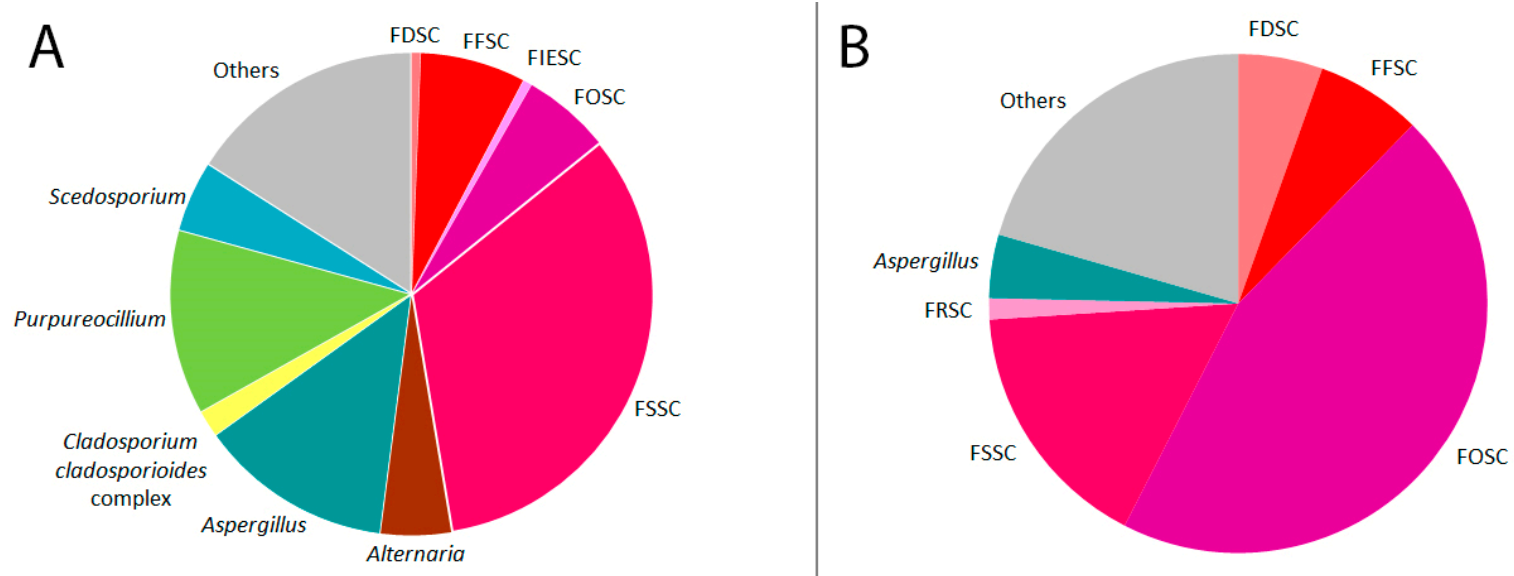

Figure 1. Taxa of filamentous fungi isolate from infected eyes (A) or eye-associated materials (B). Species complexes (sc) of Fusarium are highlighted in shades of red: Fusarium dimerum sc (FDSC), F. fujikuroi sc (FFSC), F. incarnatum-equiseti sc (FIESC), F. oxysporum sc (FOSC), F. redolens sc (FRSC), F. solani sc (FSSC). Genera that are presented by only one or two isolates are shown in the "Others" section of the pie chart.

Fusarium is also the dominant genus among isolates from eye-associated materials (75.3\%). Isolates of Aspergillus and Purpureocillum that make up high proportions of isolates from the eye are only rarely or not isolated from eye-associated materials (Table 1 , Figure 1). In Fusarium, most of the species that are commonly isolated from the eye, such as F. petroliphilum or F. solani, were also isolated from eye-associated materials in higher proportions than other species, but in numbers that were distinctly lower than those from the eye. An exception was the FOSC. In this complex, the number of isolates from eyeassociated materials was more than three times higher than the number of isolates from the eye (Table 1, Figure 1).

\subsection{Antifungal Susceptibility Testing Profiles of Eye-Infecting Fungal Species}

In total, AFST was performed for 257 strains of the 33 species that are considered to be eye pathogens, because they were repeatedly isolated from the infected eye. Strains isolated from eye samples and related materials exhibit similar profiles to strains from other sources (Table S1). The AFST profiles of the species causing eye infections differ strongly. None of the tested antifungals is effective against all species studied: species of the genera Purpureocillium, Lomentospora, Scedosporium, and Scopulariopsis show high MICs for amphotericin B; Aspergillus flavus and Purpureocillium spp. show high MICs for natamycin; while Fusarium species, especially of the FSSC, exhibit high MICs for isavuconazole, itraconazole, posaconazole, voriconazole, and caspofungin (Tables 2 and 3).

Table 2. Antifungal susceptibility of species isolated from infected eyes against amphotericin B (AMB), natamycin (NAT), and caspofungin (CAS). For the FSSC, the numbering of the phylogenetic species is given in the first bracket.

\begin{tabular}{|c|c|c|c|c|c|c|c|c|c|}
\hline \multirow[t]{2}{*}{ Species } & \multicolumn{6}{|c|}{ MICs in $\mathrm{mg} / \mathrm{L}$} & \\
\hline & $\begin{array}{l}\text { AMB } \\
\text { Range }\end{array}$ & GM & M50/M90 & $\begin{array}{l}\text { NAT } \\
\text { Range }\end{array}$ & GM & M50/M90 & $\begin{array}{l}\text { CAS } \\
\text { Range }\end{array}$ & GM & M50/M90 \\
\hline Alternaria alternata (10) & $0.125-1$ & 0.33 & $0.25 / 1$ & $2-4$ & 2.46 & $2 / 4$ & $\leq 0.06-0.5$ & 0.18 & $0.25 / 0.25$ \\
\hline Aspergillus flavus (12) & $1-4$ & 1.89 & $2 / 4$ & $>32$ & 50.8 & $>32 />32$ & $0.03-0.25$ & 0.096 & $0.125 / 0.25$ \\
\hline Aspergillus fumigatus (18) & $0.125-1$ & 0.31 & $0.25 / 1$ & $2-4$ & 2.62 & $2 / 4$ & $\leq 0.06-0.5$ & 0.15 & $0.125 / 0.25$ \\
\hline Aspergillus tubingensis (12) & $0.06-0.5$ & 0.14 & $0.125 / 0.25$ & $2-4$ & 2.83 & $2 / 4$ & $\leq 0.016-0.5$ & 0.12 & $0.125 / 0.5$ \\
\hline \multicolumn{10}{|l|}{ Fusarium } \\
\hline \multicolumn{10}{|l|}{ FDSC } \\
\hline F. dimerum (4) & $0.5-2$ & 1.19 & n.a. & $2-4$ & 3.36 & n.a. & $>8$ & 16 & n.a. \\
\hline Fusarium sp. (FDSC) (1) & 0.5 & n.a. & n.a. & 2 & n.a. & n.a. & $>8$ & n.a. & n.a. \\
\hline
\end{tabular}


Table 2. Cont.

\begin{tabular}{|c|c|c|c|c|c|c|c|c|c|}
\hline \multirow[t]{2}{*}{ Species } & \multicolumn{3}{|c|}{ MICs in $\mathrm{mg} / \mathrm{L}$} & & & \multirow[b]{2}{*}{ M50/M90 } \\
\hline & $\begin{array}{l}\text { AMB } \\
\text { Range }\end{array}$ & GM & M50/M90 & $\begin{array}{l}\text { NAT } \\
\text { Range }\end{array}$ & GM & M50/M90 & $\begin{array}{l}\text { CAS } \\
\text { Range }\end{array}$ & GM & \\
\hline FFSC & & & & & & & & & \\
\hline F. lactis (1) & 1 & n.a. & n.a. & 4 & n.a. & n.a. & 8 & n.a. & n.a. \\
\hline F. musae (3) & $1-4$ & 2 & n.a. & $2-4$ & 3.17 & n.a. & $>8$ & 16 & n.a. \\
\hline F. proliferatum (10) & $1-4$ & 1.62 & $2 / 2$ & $4-8$ & 6.96 & $8 / 8$ & $8 \rightarrow>8$ & 14.9 & $>8 />8$ \\
\hline F sacchari (2) & $1-2$ & 1.41 & n.a. & 4 & 4 & n.a. & $>8$ & 16 & n.a. \\
\hline F. verticillioides (2) & $1-2$ & 1.41 & n.a. & 4 & 4 & n.a. & $>8$ & 16 & n.a. \\
\hline $\begin{array}{l}\text { FIESC } \\
\text { F. equiseti (1) }\end{array}$ & 2 & n.a. & n.a. & 4 & 4 & n.a. & 0.25 & n.a. & n.a. \\
\hline FoSC (43) & $0.25-8$ & 1.39 & $2 / 2$ & $2-8$ & 4.41 & $4 / 8$ & $>8$ & 16 & $>8 />8$ \\
\hline $\begin{array}{l}\text { FRSC } \\
\text { F. redolens (1) }\end{array}$ & 1 & n.a. & n.a. & 4 & n.a. & n.a. & $>8$ & n.a. & n.a. \\
\hline FSSC & & & & & & & & & \\
\hline F. cyanescens (FSSC 27) (1) & 0.5 & n.a. & n.a. & 4 & n.a. & n.a. & $>8$ & n.a. & n.a. \\
\hline F. falciforme (FSSC 3+4) (5) & $1-2$ & 1.15 & n.a. & 8 & n.a. & n.a. & $>8$ & n.a. & n.a. \\
\hline F. ferrugineum (FSSC 28) (1) & 1 & n.a. & n.a. & 4 & n.a. & n.a. & $>8$ & n.a. & n.a. \\
\hline F. keratoplasticum (FSSC 2) (14) & $1-4$ & 2.56 & $2 / 4$ & $4-8$ & 5.12 & $4 / 8$ & $>8$ & 16 & $>8 />8$ \\
\hline F. metavorans (FSSC 6) (2) & $1-8$ & 2.83 & n.a. & $4-8$ & 5.66 & n.a. & $8 \rightarrow>8$ & 11.3 & n.a. \\
\hline F. pisi (FSSC 11) (1) & 2 & n.a. & n.a. & 8 & n.a. & n.a. & $>8$ & n.a. & n.a. \\
\hline F. petroliphilum (FSSC 1) (27) & $0.5-8$ & 1.29 & $2 / 2$ & $2-8$ & 5.04 & $4 / 8$ & $8->8$ & 15.5 & $>8 />8$ \\
\hline F. solani (FSSC 5) (21) & $0.5-8$ & 1.39 & $1 / 2$ & $4-32$ & 9.59 & $8 / 16$ & $>8$ & 16 & $>8 />8$ \\
\hline F. bostrycoides(FSSC 25+35) (3) & 1 & 1 & n.a. & $2-4$ & 2.83 & n.a. & $>8$ & 16 & n.a. \\
\hline F. stercicola (FSSC 44) (1) & 2 & n.a. & n.a. & 4 & n.a. & n.a. & $>8$ & n.a. & n.a. \\
\hline F. tonkinense (FSSC 9) (2) & $2-4$ & 2.83 & n.a. & 48 & 5.66 & n.a. & $>8$ & 16 & n.a. \\
\hline Purpureocillium lilacinum (15) & $>16$ & 32 & $>8 />8$ & $>32$ & 64 & $>32 />32$ & $0.125->8$ & 0.87 & $1 />8$ \\
\hline Purpureocillium sodanum (7) & $>16$ & 32 & n.a. & $>32$ & 64 & n.a. & $0.25->8$ & 0.91 & n.a. \\
\hline Lomentospora prolificans (11) & $4->16$ & 26.5 & $>8 />8$ & $2-8$ & 5.84 & $8 / 8$ & $1-8$ & 2.83 & $4 / 8$ \\
\hline Scedosporium apiospermum (17) & $1->16$ & 6.26 & $8 />8$ & $2-4$ & 2.55 & $2 / 4$ & $0.125-2$ & 0.82 & $1 / 1$ \\
\hline Scedosporium aurantiacum (1) & $>16$ & n.a. & n.a. & 2 & n.a. & n.a. & 4 & n.a. & n.a. \\
\hline Scedosporium dehoogii (4) & $8->16$ & 13.5 & n.a. & 2 & 2 & n.a. & $1 \rightarrow>8$ & 5.66 & n.a. \\
\hline Scopulariopsis brevicaulis (2) & $8->16$ & 16 & n.a. & 4 & 4 & n.a. & 0.5 & 0.5 & n.a. \\
\hline Tintelnotia destructans (3) & $0.5-1$ & 0.63 & n.a. & $1-2$ & 1.41 & n.a. & $\leq 0.016-0.25$ & 0.06 & n.a. \\
\hline
\end{tabular}

Amphotericin B and natamycin are both polyene antifungals. However, high MICs for amphotericin B did not necessarily correspond to high natamycin MICs in all taxa. Species of Scedosporium, Lomentospora prolificans, and Scopulariopsis brevicaulis have high MICs for amphotericin B but low MICs for natamycin (Table 2). In contrast, Purpureocillium spp. show high MICs for amphotericin B and for natamycin (Table 2). While this may suggest differential activity of the two agents, it remains unclear how this translates into clinical treatment response. 
Table 3. Antifungal susceptibility of species isolated from infected eyes against isavuconazole (ISA), itraconazole (ITZ), posaconazole (PCZ), and voriconazole (VCZ). For the FSSC, the numbering of the phylogenetic species is given in the first bracket.

\begin{tabular}{|c|c|c|c|c|c|c|c|c|c|c|c|c|}
\hline \multirow[t]{3}{*}{ Species } & \multicolumn{6}{|c|}{ MICs in mg/L } & \multirow{2}{*}{\multicolumn{3}{|c|}{ PCZ }} & \\
\hline & ISA & & & ITZ & & & & & & $\mathrm{VCZ}$ & & \\
\hline & Range & GM & M50/M90 & Range & GM & M50/M90 & Range & GM & M50/M90 & Range & GM & M50/M90 \\
\hline A. alternata (10) & $0.5-16$ & 3.03 & $4 / 8$ & $0.25-16$ & 0.93 & $0.5 / 1$ & $\leq 0.016-0.5$ & 0.10 & $0.125 / 0.5$ & $0.5-4$ & 1.74 & $2 / 2$ \\
\hline A. flavus (12) & $0.5-2$ & 0.75 & $0.5 / 2$ & $0.25-2$ & 0.47 & $0.5 / 1$ & $0.125-0.5$ & 0.177 & $0.125 / 0.5$ & $0.5-1$ & 0.59 & $0.5 / 1$ \\
\hline A. fumigatus (18) & $0.125-8$ & 0.71 & $0.5 / 8$ & $0.125->8$ & 0.68 & $0.5 />8$ & $\leq 0.016-2$ & 0.06 & $0.06 / 0.5$ & $0.25-4$ & 0.58 & $0.5 / 2$ \\
\hline A. tubingensis (12) & $1-8$ & 2.92 & $4 / 4$ & $1->8$ & 4 & $2 />8$ & $0.06-1$ & 0.26 & $0.25 / 0.5$ & $0.5-2$ & 1.19 & $1 / 2$ \\
\hline \multicolumn{13}{|l|}{ Fusarium } \\
\hline \multicolumn{13}{|l|}{ FDSC } \\
\hline F. dimerum (4) & $>8$ & 16 & n.a. & $>8$ & 16 & n.a. & $>8$ & 16 & n.a. & $4-8$ & 6.73 & n.a. \\
\hline Fusarium sp. (FDSC) (1) & 8 & n.a. & n.a. & $>8$ & n.a. & n.a. & $>8$ & n.a. & n.a. & 4 & n.a. & n.a. \\
\hline \multicolumn{13}{|l|}{ FFSC } \\
\hline F. lactis (1) & $>8$ & n.a. & n.a. & $>8$ & n.a. & n.a. & $>8$ & n.a. & n.a. & 8 & n.a. & n.a. \\
\hline F. musae (3) & 4 & 4 & n.a. & $>8$ & 16 & n.a. & $1-2$ & 1.26 & n.a. & $2-4$ & 2.52 & n.a. \\
\hline F. proliferatum (10) & $>8$ & 16 & $>8 />8$ & $>8$ & 16 & n.a. & $4->8$ & 6.06 & $>8 />8$ & $4-8$ & 5.66 & $4 / 8$ \\
\hline F sacchari (2) & $2-4$ & 2.83 & n.a. & $>8$ & 11 & n.a. & 0.5 & 0.5 & n.a. & 2 & 2 & n.a. \\
\hline F. verticillioides (2) & $2-4$ & 2.83 & n.a. & $2->8$ & 5.7 & n.a. & $0.25-1$ & 0.5 & n.a. & $1-2$ & 1.41 & n.a. \\
\hline \multicolumn{13}{|l|}{ FIESC } \\
\hline F. equiseti (1) & 2 & n.a. & n.a. & 1 & n.a. & n.a. & 0.5 & n.a. & n.a. & 1 & n.a. & n.a. \\
\hline F0SC (43) & $4->8$ & 13.0 & $>8 />8$ & $>8$ & 16 & $>8 />8$ & $1->8$ & 12.77 & $>8 />8$ & $2->8$ & 4.78 & $4 />8$ \\
\hline \multicolumn{13}{|l|}{ FRSC } \\
\hline F. redolens (1) & $>8$ & n.a. & n.a. & $>8$ & n.a. & n.a. & $>8$ & n.a. & & 4 & n.a. & n.a. \\
\hline \multicolumn{13}{|l|}{ FSSC } \\
\hline F. cyanescens (FSSC 27) (1) & $>8$ & n.a. & n.a. & $>8$ & n.a. & n.a. & $>8$ & n.a. & n.a. & $>8$ & n.a. & n.a. \\
\hline F. falciforme (FSSC 3+4) (5) & $>8$ & 16 & n.a. & $>8$ & 16 & $>8 />8$ & $>8$ & 16 & n.a. & $>8$ & 16 & n.a. \\
\hline F. ferrugineum (FSSC 28) (1) & $>8$ & n.a. & n.a. & $>8$ & n.a. & n.a. & $>8$ & n.a. & n.a. & 4 & n.a. & n.a. \\
\hline $\begin{array}{l}\text { F. keratoplasticum } \\
\text { (FSSC 2) (14) }\end{array}$ & $>8$ & 16 & $>8 />8$ & $>8$ & 16 & $>8 />8$ & $>8$ & 16 & $>8 />8$ & $4->8$ & 12.50 & $>8 />8$ \\
\hline F. metavorans (FSSC 6) (2) & $8->8$ & 11.3 & n.a. & $8->8$ & 11 & n.a. & $8->8$ & 11.3 & n.a. & $4-8$ & 5.66 & n.a. \\
\hline F. pisi (FSSC 11) (1) & $>8$ & n.a & n.a. & $>8$ & n.a & n.a. & $>8$ & n.a & n.a. & $>8$ & n.a & n.a. \\
\hline F. petroliphilum (FSSC 1) (27) & $>8$ & 16 & $>8 />8$ & $>8$ & 16 & $>8 />8$ & $>8$ & 16 & $>8 />8$ & $4->8$ & 14.4 & $>8 />8$ \\
\hline F. solani (FSSC 5) (21) & $>8$ & 16 & $>8 />8$ & $>8$ & 16 & $>8 />8$ & $>8$ & 15.5 & $>8 />8$ & $4->8$ & 12.7 & $>8 />8$ \\
\hline $\begin{array}{l}\text { F. bostrycoides (FSSC 25+35) } \\
\text { (3) }\end{array}$ & $>8$ & 16 & n.a. & $>8$ & 16 & n.a. & $>8$ & 16 & n.a. & $1->8$ & 4 & n.a. \\
\hline F. stercicola (FSSC 44) (1) & $>8$ & n.a & n.a. & $>8$ & n.a & n.a. & $>8$ & n.a & n.a. & $>8$ & n.a & n.a. \\
\hline F. tonkinense (FSSC 9) (2) & $>8$ & 16 & n.a. & $>8$ & 16 & n.a. & $>8$ & 16 & n.a. & $>8$ & 16 & n.a. \\
\hline P. lilacinum (15) & $0.06-2$ & 0.34 & $0.5 / 1$ & $1->8$ & 5.79 & $>8 />8$ & $0.06-1$ & 0.16 & $0.125 / 0.5$ & $0.125-0.5$ & 0.24 & $0.25 / 0.5$ \\
\hline P. sodanum (7) & $0.125-1$ & 0.35 & n.a. & $2->8$ & \#\#\# & n.a. & $0.06-0.5$ & 0.15 & n.a. & $0.125-0.5$ & 0.25 & n.a. \\
\hline L. prolificans (11) & $4->8$ & 12.40 & $>8 />8$ & $>8$ & 16 & $>8 />8$ & $>8$ & 16 & $>8 />8$ & $4->8$ & 13.2 & $>8 />8$ \\
\hline S. apiospermum (17) & $>8$ & 11.60 & $>8 />8$ & $2-8$ & 12 & n.a. & $1->8$ & 5.77 & $4 />8$ & $0.5-1$ & 0.75 & $1 / 1$ \\
\hline S. aurantiacum (1) & 8 & n.a & n.a. & $>8$ & n.a & n.a. & 2 & n.a & n.a & 0.5 & n.a & n.a \\
\hline S. dehoogii (4) & $2-8$ & 4 & n.a. & $1->8$ & 2 & n.a. & $0.25-2$ & 0.71 & n.a & $0.25-0.5$ & 0.42 & n.a \\
\hline Scopulariopsis brevicaulis (2) & $>8$ & 16.00 & n.a. & $>8$ & 16 & n.a. & $>8$ & 16 & n.a & $>8$ & 16 & n.a \\
\hline Tintelnotia destructans (3) & $4-8$ & 6.35 & n.a. & $0.25-0.5$ & 0.40 & n.a. & $0.06-0.125$ & 0.10 & n.a & $0.5-1$ & 0.79 & n.a \\
\hline
\end{tabular}

Within the genus Fusarium, the studied species complexes did not differ regarding their susceptibility to amphotericin B, natamycin, itraconazole, and caspofungin (Tables 2 and 3). In vitro, amphotericin B was the most effective drug. The MICs for natamycin ranged between 2 and $8 \mathrm{mg} / \mathrm{L}$, except for F. solani, with natamycin MICs of up to $32 \mathrm{mg} / \mathrm{L}$. Species of the FSSC had high MICs for the azoles tested (ISA, ITZ, PCZ, VCZ), with the lowest MICs found for voriconazole. Slightly lower MICs for voriconazole were found in the FOSC. The species of FFSC possessed specific profiles-F. musae, F. sacchari, and F. verticillioides were more susceptible to azoles, especially posaconazole and voriconazole, while F. proliferatum and F. lactis had MICs similar to the FSSC (Tables 2 and 3).

Purpureocillium lilacinum and P. sodanum, as well as Tintelnotia destructans, had characteristic AFS profiles for azoles. The two Purpureocillium species showed high MICs 
for itraconazole but low MICs for isavuconazole, posaconazole, and voriconazole, while Tintelnotia destructans exhibited high MICs for isavuconazole but low MICs for itraconazole, posaconazole, and voriconazole.

All three studied species of Scedosporium showed low MICs for voriconazole, in contrast to the closely related Lomentospora prolificans, which showed high MICs for voriconazole as well (Tables 2 and 3).

\section{Discussion}

This study presents the first spectrum of filamentous fungi isolated from infected eyes that are molecularly identified by the use of the ID markers established in the respective genera. Sample submission to the NRZMyk is non-systematic and likely biased towards clinically relevant isolates and isolates that are not easily identified.

Fifty-two species were isolated from the eye and eye-associated materials, while 14 species were restricted to eye-associated materials only. Chaetomium anastomosans, Coprinellus domesticus (syn. Hormographiella verticillata), Eppicoccum mezzettii, Fusarium stercicola, Lecanicillium coprophilum, Penicillium crustosum, Petriella setifera, Purpureocillium sodanum, Rhinocladiella similis, and Sarocladium spinificis have not been reported to cause eye infections. Chaetomium anastomosans (sibling species of C. globosum) [30] and Purpureocillium sodanum (sibling species of P. lilacinum) [31] are recently described species, cases of which might have previously been assigned to their sibling species. Other species such as Aspergillus cibarius [32], A. udagawae [33], Fusarium bostrycoides (as FSSC 25), F. tonkinense (as FSSC 9) [9], Penicillium rubens [34], and Scedosporium dehoogii [35] have been reported only once before in connection with eye infections.

Among all filamentous fungi associated with eye infections, Fusarium is the dominating genus, at nearly $47.3 \%$. In the genus Fusarium, species of the FSSC (F. petroliphilum, F. keratoplasticum, and F. solani) are most frequently isolated from the infected eye, in agreement with former studies [9,36-39]. Studies in France and the Netherlands found higher proportions of the FFSC (47\%) and the FOSC (41\%) [40] or the FOSC (24.7\%) [10], respectively. FOSC from contact lenses and their cleaning solution suggests that this species is a frequent contaminant of eye-associated materials, which is supported by a previous case study [9]. Some studies have shown a lower pathogenic potential of the FOSC compared to the FSSC [41-43]. On the other hand, this observation could mean that the cultivation of the FOSC from infected tissue fails more often, e.g., because its viability is more affected by antifungal treatment.

Compared to species of Aspergillus (5.5\%) and Purpureocillium (0\%), Fusarium spp. were more frequently isolated from contact lenses or their cleaning solutions $(75.3 \%$ in total: FOSC 45.2\%, remaining Fusarium species 30.1\%; Table 1, Figure 1). One reason could be that Aspergillus spp. and Purpureocillium spp. grow better from eye samples, making isolations from eye-associated materials unnecessary. Another reason could be that the antimicrobial agents of the cleaning solutions are less effective against Fusarium species; thus, the reduced activity of alexidine in contact lens cleaning solutions after heating caused a worldwide outbreak of Fusarium keratitis, while cases of keratitis caused by other fungal genera did not increase markedly $[44,45]$.

Other important eye-infecting genera are Aspergillus (13.0\%), Purpureocillium (12.4\%), Alternaria (4.7\%), and Scedosporium (4.7\%). Strikingly, dematiaceous genera such as $\mathrm{Al}$ ternaria and Cladosporium contribute to a smaller extent compared to studies of tropical and subtropical regions with more intensive UV radiation [2,12,36,46-50]. Dematiaceous genera such as Bipolaris and Curvularia, which are important eye pathogens in the tropics and subtropics, were not sent to the NRZMyk.

The high proportion of Purpureocillium species in eye infections in Germany is remarkable. Purpureocillium lilacinum used to be named Paecilomyces lilacinus. Paecilomyces spp. are mentioned as causative agents in several studies of fungal keratitis $[46,48,49]$, but the percentage of the Purpureocillium species included is unknown. In the Assam region of North India, Purpureocillium lilacinum is causative in $1.6 \%$ of fungal keratitis cases [51], 
while in the USA it accounts for 4.4\% [52]. One of the major risk factors for infections by P. lilacinum is the use of contact lenses [52,53], which might explain its high proportion in Germany, where wearing contact lenses is popular [54]. Purpureocillium lilacinum and its sibling species $P$. sodanum [31] are morphologically similar but their ITS sequences differ only by a single base pair. As a consequence, it is more likely that $P$. sodanum has been overlooked than that it is emerging.

The important keratitis-causing fungi in Germany show diverse antifungal susceptibility profiles and include taxa with high MICs for all antifungals tested. Amphotericin B was the antifungal agent with the lowest MICs for Fusarium spp. and with low MICs for several other species (Tables 2 and 3); however, eye pathogens such as Purpureocillium spp., Lomentospora prolificans, Scedosporium spp., and Scopulariopsis brevicaulis exhibit high amphotericin B MICs, which is in agreement with previous studies [28,55-59]. Natamycin MICs are usually higher compared to amphotericin B MICs, but isolates with natamycin MICs of $\leq 16 \mathrm{mg} / \mathrm{L}$ are considered susceptible because this concentration is reached in the eye during therapy $[60,61]$. The ineffectiveness of natamycin for Aspergillus flavus and Purpureocillium spp. has been reported previously [52,55].

Some of the Fusarium solani strains tested in our study had reduced susceptibility for natamycin (MIC $>16 \mathrm{mg} / \mathrm{L}$ ). Although members of the FSSC exhibit similar AFS profiles, we found MIC $>16 \mathrm{mg} / \mathrm{L}$ only for this species of the FSSC. In a Dutch study, MICs of 16 $\mathrm{mg} / \mathrm{L}$ for some strains of $F$. solani and F. falciforme were observed [62]. An Indian study found natamycin MICs for the FSSC ranging between 8-128 mg/L [55]. To date, we do not know if these results of in vitro tests have an impact on the outcome of $F$. solani infections, but in keratitis cases caused by this species that do not respond to natamycin, a switch of the therapeutic agent should be considered.

The genera included in our study showed different susceptibilities concerning the polyenes-Purpureocillium spp. were not susceptible to amphotericin B or natamycin, Aspergillus flavus showed reduced MICs for amphotericin B but high MICs for natamycin and Scedosporium spp., Lomentospora prolificans and Scopulariopsis brevicaulis exhibited normal MICs for natamycin but high MICs for amphotericin B. These results are in concordance with the finding that natamycin has a different mode of action. As all polyene drugs, natamycin binds to ergosterol; however, in contrast to amphotericin $B$, it does not change the permeability of the plasma membrane, resulting in the leakage of essential components; rather, it impairs the membrane fusion $[63,64]$.

The susceptibility levels for azoles determined in this study show differences among the species complexes that are in agreement with former studies [65-67], with high MICs for all azoles, including voriconazole in the FSSC; slightly lower MICs for voriconazole in the FOSC; and lower MICs for isavuconazole, posaconazole and voriconazole for most of the FFSC species. One exception to this is Fusarium proliferatum, which also had high MICs for isavuconazole, posaconazole, and voriconazole, although it belongs to the FFSC. By using the CLSI protocol, lower posaconazole MICs were found for this species [26].

In conclusion, in Germany the predominant filamentous fungi infecting eyes belong to the genera Fusarium, Aspergillus, Purpureocillium, Alternaria, and Scedosporium. Differences in their AFS profiles, which include high MICs for all important antifungals in keratitis treatment (natamycin, amphotericin B, voriconazole), favor combined therapy and underline the importance of the identification of the aetiological agent. Some strains of Fusarium solani exhibited natamycin MICs $>16 \mathrm{mg} / \mathrm{L}$. Although amphotericin B and natamycin are both polyenes, the levels of MIC values of amphotericin B of a certain species are not predictive of MIC values of natamycin, and vice versa.

Supplementary Materials: The following are available online at https:/ /www.mdpi.com/article/10 .3390/jof7070511/s1, Table S1: Strains studied. their strain numbers. sources. GenBank accession numbers. and antifungal susceptibilities for amphotericin B (AMB). natamycin (NAT). isavuconazole (ISA). itraconazole (ITZ). posaconazole (PCZ). voriconazole (VCZ) and caspofungin (CAS). Strains from the same patient are marked. Table S2: Markers amplified for the identification of eye infecting filamentous fungi, primers and annealing temperatures used for PCR. 
Author Contributions: Conceptualization, G.W. and O.K.; methodology, G.W. and K.K.; validation, G.W. and A.Z.; formal analysis, G.W. and A.Z.; data curation, G.W. and K.K.; writing-original draft preparation, G.W.; writing-review and editing, G.W., A.Z., J.T., K.K., M.v.L.-T., M.R., D.K., G.G., O.K.; funding acquisition, O.K., D.K. All authors have read and agreed to the published version of the manuscript.

Funding: This work was supported by the Interdisciplinary Center for Clinical Research (IZKF) at the University Hospital Würzburg (grant numbers D-420 to D.K. and O.K.). The work of the NRZMyk is supported by the Robert Koch Institute from funds provided by the German Ministry of Health (grant-No. 1369-240).

Institutional Review Board Statement: Not applicable.

Informed Consent Statement: Not applicable.

Data Availability Statement: All raw data can be found in Table S1.

Acknowledgments: For their excellent technical assistance, we thank Philipp Hupel, Carmen Karkowski, Grit Mrotzek, and Christiane Weigel. We are also grateful to Basilea, MDS, and Pfizer, Inc. for providing the drugs for the susceptibility testing. Furthermore, we thank our colleagues in hospitals and laboratories for sending their strains.

Conflicts of Interest: M.v.L.-T. received honoraria and/or research support from Celgene, Gilead, Chugai, Janssen, Novartis, Amgen, Takeda, BMS, Medac, Oncopeptides, MSD, CDDF, abbvie, AstraZeneca, Pfizer Novartis, Gilead, Celgene, Oncopeptides, 4DPharma, Shionogi. G.G. received honoraria and/or research support from Santen, Chiesi, Novartis, Med1Ventures, Akceso Advisors, Alcon, Dompé, Sun Pharma, and Shire. O.K. received honoraria and/or research support (reagents) from Pfizer, Gilead, MSD, Basilea, Virotech, and Fujifilm Wako.

\section{References}

1. Brown, L.; Leck, A.K.; Gichangi, M.; Burton, M.J.; Denning, D.W. The global incidence and diagnosis of fungal keratitis. Lancet Infect. Dis. 2021, 21, e49-e57. [CrossRef]

2. Gopinathan, U.; Garg, P.; Fernandes, M.; Sharma, S.; Athmanathan, S.; Rao, G.N. The epidemiological features and laboratory results of fungal keratitis: A 10-year review at a referral eye care center in South India. Cornea 2002, 21, 555-559. [CrossRef]

3. Bongomin, F.; Gago, S.; Oladele, R.O.; Denning, D.W. Global and multi-national prevalence of fungal diseases-estimate precision. J. Fungi 2017, 3, 57. [CrossRef]

4. Cheng, K.H.; Leung, S.L.; Hoekman, H.W.; Beekhuis, W.H.; Mulder, P.G.H.; Geerards, A.J.M.; Kijlstra, A. Incidence of contactlens-associated microbial keratitis and its related morbidity. Lancet 1999, 354, 181-185. [CrossRef]

5. Stapleton, F.; Keay, L.; Edwards, K.; Naduvilath, T.; Dart, J.K.; Brian, G.; Holden, B.A. The incidence of contact lens-related microbial keratitis in Australia. Ophthalmology 2008, 115, 1655-1662. [CrossRef] [PubMed]

6. Jurkunas, U.; Behlau, I.; Colby, K. Fungal keratitis: Changing pathogens and risk factors. Cornea 2009, 28, 638-643. [CrossRef]

7. Nielsen, S.E.; Nielsen, E.; Julian, H.O.; Lindegaard, J.; Hojgaard, K.; Ivarsen, A.; Hjortdal, J.; Heegaard, S. Incidence and clinical characteristics of fungal keratitis in a Danish population from 2000 to 2013. Acta Ophthalmol. 2015, 93, 54-58. [CrossRef]

8. Ong, H.S.; Fung, S.S.M.; Macleod, D.; Dart, J.K.G.; Tuft, S.J.; Burton, M.J. Altered patterns of fungal keratitis at a London ophthalmic referral hospital: An eight-year retrospective observational study. Am. J. Ophthalmol. 2016, 168, 227-236. [CrossRef]

9. Walther, G.; Stasch, S.; Kaerger, K.; Hamprecht, A.; Roth, M.; Cornely, O.A.; Geerling, G.; Mackenzie, C.R.; Kurzai, O.; von Lilienfeld-Toal, M. Fusarium keratitis in Germany. J. Clin. Microbiol. 2017, 55, 2983-2995. [CrossRef]

10. Oliveira Dos Santos, C.; Kolwijck, E.; van Rooij, J.; Stoutenbeek, R.; Visser, N.; Cheng, Y.Y.; Santana, N.T.Y.; Verweij, P.E.; Eggink, C.A. Epidemiology and clinical management of Fusarium keratitis in the Netherlands, 2005-2016. Front. Cell. Infect. Microbiol. 2020, 10, 133. [CrossRef] [PubMed]

11. Thomas, P.A.; Kaliamurthy, J. Mycotic keratitis: Epidemiology, diagnosis and management. Clin. Microbiol. Infect. 2013, 19, 210-220. [CrossRef]

12. Kredics, L.; Narendran, V.; Shobana, C.S.; Vagvolgyi, C.; Manikandan, P.; Indo-Hungarian Fungal Keratitis Working Group. Filamentous fungal infections of the cornea: A global overview of epidemiology and drug sensitivity. Mycoses 2015, 58, 243-260. [CrossRef]

13. Hoffman, J.J.; Burton, M.J.; Leck, A. Mycotic keratitis—A global threat from the filamentous fungi. J. Fungi 2021, 7, 273. [CrossRef] [PubMed]

14. Roth, M.; Daas, L.; Renner-Wilde, A.; Cvetkova-Fischer, N.; Saeger, M.; Herwig-Carl, M.; Matthaei, M.; Fekete, A.; Kakkassery, V.; Walther, G.; et al. The German keratomycosis registry: Initial results of a multicenter survey. Ophthalmologe 2019, 116, 957-966. [CrossRef] [PubMed] 
15. Short, D.P.; O’Donnell, K.; Thrane, U.; Nielsen, K.F.; Zhang, N.; Juba, J.H.; Geiser, D.M. Phylogenetic relationships among members of the Fusarium solani species complex in human infections and the descriptions of F. keratoplasticum sp. nov. and $F$. petroliphilum stat. nov. Fungal Genet. Biol. 2013, 53, 59-70. [CrossRef] [PubMed]

16. Sandoval-Denis, M.; Lombard, L.; Crous, P.W. Back to the roots: A reappraisal of Neocosmospora. Persoonia 2019, 43, 90-185. [CrossRef]

17. Jurjevic, Z.; Peterson, S.W.; Horn, B.W. Aspergillus section Versicolores: Nine new species and multilocus DNA sequence based phylogeny. IMA Fungus 2012, 3, 59-79. [CrossRef]

18. Nováková, A.; Hubka, V.; Dudová, Z.; Matsuzawa, T.; Kubátová, A.; Yaguchi, T.; Kolařík, M. New species in Aspergillus section Fumigati from reclamation sites in Wyoming (U.S.A.) and revision of A. viridinutans complex. Fungal Divers. 2013, 64, 253-274. [CrossRef]

19. Frisvad, J.C.; Hubka, V.; Ezekiel, C.N.; Hong, S.B.; Nováková, A.; Chen, A.J.; Arzanlou, M.; Larsen, T.O.; Sklenář, F.; Mahakarnchanakul, W.; et al. Taxonomy of Aspergillus section Flavi and their production of aflatoxins, ochratoxins and other mycotoxins. Stud. Mycol. 2019, 93, 1-63. [CrossRef]

20. Schoch, C.L.; Seifert, K.A.; Huhndorf, S.; Robert, V.; Spouge, J.L.; Levesque, C.A.; Chen, W.; Fungal Barcoding Consortium. Nuclear ribosomal internal transcribed spacer (ITS) region as a universal DNA barcode marker for Fungi. Proc. Natl. Acad. Sci. USA 2012, 109, 6241-6246. [CrossRef]

21. Samson, R.A.; Noonim, P.; Meijer, M.; Houbraken, J.; Frisvad, J.C.; Varga, J. Diagnostic tools to identify black aspergilli. Stud. Mycol 2007, 59, 129-145. [CrossRef]

22. Kebabci, N.; van Diepeningen, A.D.; Ener, B.; Ersal, T.; Meijer, M.; Al-Hatmi, A.M.; Ozkocaman, V.; Ursavas, A.; Cetinoglu, E.D.; Akalin, H. Fatal breakthrough infection with Fusarium andiyazi: New multi-resistant aetiological agent cross-reacting with Aspergillus galactomannan enzyme immunoassay. Mycoses 2014, 57, 249-255. [CrossRef] [PubMed]

23. Geiser, D.M.; Al-Hatmi, A.; Aoki, T.; Arie, T.; Balmas, V.; Barnes, I.; Bergstrom, G.C.; Bhattacharyya, M.K.K.; Blomquist, C.L.; Bowden, R.; et al. Phylogenomic analysis of a $55.1 \mathrm{~kb} 19$-gene dataset resolves a monophyletic Fusarium that includes the Fusarium solani species complex. Phytopathology 2020. [CrossRef] [PubMed]

24. Lombard, L.; van der Merwe, N.A.; Groenewald, J.Z.; Crous, P.W. Generic concepts in Nectriaceae. Stud. Mycol. 2015, 80, 189-245. [CrossRef] [PubMed]

25. FlorCruz, N.V.; Evans, J.R. Medical interventions for fungal keratitis. Cochrane Database Syst. Rev. 2015, CD004241. [CrossRef] [PubMed]

26. Al-Hatmi, A.M.; van Diepeningen, A.D.; Curfs-Breuker, I.; de Hoog, G.S.; Meis, J.F. Specific antifungal susceptibility profiles of opportunists in the Fusarium fujikuroi complex. J. Antimicrob Chemother 2015, 70, 1068-1071. [CrossRef]

27. De Hoog, G.S.; Guarro, J.; Gene, J.; Ahmed, A.M.S.; Al-Hatmi, A.; Figueras, M.J.; Vitale, R.G. Atlas of Clinical Fungi; Centraalbureau voor Schimmelcultures (CBS): Utrecht, The Netherlands, 2020; p. 1599.

28. Lackner, M.; de Hoog, G.S.; Verweij, P.E.; Najafzadeh, M.J.; Curfs-Breuker, I.; Klaassen, C.H.; Meis, J.F. Species-specific antifungal susceptibility patterns of Scedosporium and Pseudallescheria species. Antimicrob. Agents Chemother. 2012, 56, 2635-2642. [CrossRef]

29. Arendrup, M.C.; Meletiadis, J.; Mouton, J.W.; Lagrou, K.; Hamal, P.; Guinea, J. EUCAST Definitive Document, E.DEF 9.3.2: Method for the Determination of Broth Dilution Minimum Inhibitory Concentrations of Antifungal Agents for Conidia Forming Moulds. Available online: https://www.eucast.org/astoffungi/methodsinantifungalsusceptibilitytesting/ast_of_moulds/ (accessed on 15 June 2020).

30. Raza, M.; Zhang, Z.-F.; Hyde, K.D.; Diao, Y.-Z.; Cai, L. Culturable plant pathogenic fungi associated with sugarcane in southern China. Fungal Divers. 2019, 99, 1-104. [CrossRef]

31. Hyde, K.D.; Hongsanan, S.; Jeewon, R.; Bhat, D.J.; McKenzie, E.H.C.; Jones, E.B.G.; Phookamsak, R.; Ariyawansa, H.A.; Boonmee, S.; Zhao, Q.; et al. Fungal diversity notes 367-490: Taxonomic and phylogenetic contributions to fungal taxa. Fungal Divers. 2016, 80, 1-270. [CrossRef]

32. Hayashi, Y.; Eguchi, H.; Toibana, T.; Mitamura, Y.; Yaguchi, T. Polymicrobial sclerokeratitis caused by Scedosporium apiospermum and Aspergillus cibarius. Cornea 2014, 33, 875-877. [CrossRef] [PubMed]

33. Cheung, N.N.; Cheng, Y.Y.Y.; van Duinen, S.G.; Houbraken, J.; Verweij, P.E.; Gooskens, J. Contact lens-related fungal keratitis. Lancet Infect. Dis. 2020, 20, s1473-s3099. [CrossRef]

34. Guevara-Suarez, M.; Sutton, D.A.; Cano-Lira, J.F.; Garcia, D.; Martin-Vicente, A.; Wiederhold, N.; Guarro, J.; Gene, J. Identification and antifungal susceptibility of Penicillium-like fungi from clinical samples in the United States. J. Clin. Microbiol. 2016, 54, 2155-2161. [CrossRef]

35. Abrantes, R.A.; Refojo, N.; Hevia, A.I.; Fernandez, J.; Isla, G.; Cordoba, S.; Davalos, M.F.; Lubovich, S.; Maldonado, I.; Davel, G.O.; et al. Scedosporium spp. from clinical setting in Argentina, with the proposal of the new pathogenic species Scedosporium americanum. J. Fungi 2021, 7, 160. [CrossRef]

36. Ghosh, A.K.; Gupta, A.; Rudramurthy, S.M.; Paul, S.; Hallur, V.K.; Chakrabarti, A. Fungal keratitis in North India: Spectrum of agents, risk factors and treatment. Mycopathologia 2016, 181, 843-850. [CrossRef]

37. Xie, L.; Zhong, W.; Shi, W.; Sun, S. Spectrum of fungal keratitis in north China. Ophthalmology 2006, 113, 1943-1948. [CrossRef] [PubMed]

38. Wang, L.; Sun, S.; Jing, Y.; Han, L.; Zhang, H.; Yue, J. Spectrum of fungal keratitis in central China. Clin. Exp. Ophthalmol. 2009, 37, 763-771. [CrossRef] [PubMed] 
39. Cheikhrouhou, F.; Makni, F.; Neji, S.; Trigui, A.; Sellami, H.; Trabelsi, H.; Guidara, R.; Fki, J.; Ayadi, A. Epidemiological profile of fungal keratitis in Sfax (Tunisia). J. Mycol. Med. 2014, 24, 308-312. [CrossRef]

40. Gaujoux, T.; Chatel, M.A.; Chaumeil, C.; Laroche, L.; Borderie, V.M. Outbreak of contact lens-related Fusarium keratitis in France. Cornea 2008, 27, 1018-1021. [CrossRef] [PubMed]

41. Ahearn, D.G.; Zhang, S.; Stulting, R.D.; Schwam, B.L.; Simmons, R.B.; Ward, M.A.; Pierce, G.E.; Crow, S.A., Jr. Fusarium keratitis and contact lens wear: Facts and speculations. Med. Mycol. 2008, 46, 397-410. [CrossRef]

42. Mayayo, E.; Pujol, I.; Guarro, J. Experimental pathogenicity of four opportunist Fusarium species in a murine model. J. Med. Microbiol. 1999, 48, 363-366. [CrossRef]

43. Zhang, S.; Ahearn, D.G.; Stulting, R.D.; Schwam, B.L.; Simmons, R.B.; Pierce, G.E.; Crow, S.A., Jr. Differences among strains of the Fusarium oxysporum-F. solani complexes in their penetration of hydrogel contact lenses and subsequent susceptibility to multipurpose contact lens disinfection solutions. Cornea 2007, 26, 1249-1254. [CrossRef]

44. Chang, D.C.; Grant, G.B.; O’Donnell, K.; Wannemuehler, K.A.; Noble-Wang, J.; Rao, C.Y.; Jacobson, L.M.; Crowell, C.S.; Sneed, R.S.; Lewis, F.M.T.; et al. Multistate outbreak of Fusarium keratitis associated with use of a contact lens solution. JAMA 2006, 296. [CrossRef]

45. Bullock, J.D.; Warwar, R.E.; Elder, B.L.; Khamis, H.J. Microbiological investigations of ReNu plastic bottles and the 2004 to 2006 ReNu with moistureLoc-related worldwide Fusarium keratitis event. Eye Contact Lens. 2016, 42, 147-152. [CrossRef]

46. Leck, A.K.; Thomas, P.A.; Hagan, M.; Kaliamurthy, J.; Ackuaku, E.; John, M.; Newman, M.J.; Codjoe, F.S.; Opintan, J.A.; Kalavathy, C.M.; et al. Aetiology of suppurative corneal ulcers in Ghana and south India, and epidemiology of fungal keratitis. Br. J. Ophthalmol. 2002, 86, 1211-1215. [CrossRef]

47. Chowdhary, A.; Singh, K. Spectrum of fungal keratitis in North India. Cornea 2005, 24, 8-15. [CrossRef] [PubMed]

48. Ho, J.W.; Fernandez, M.M.; Rebong, R.A.; Carlson, A.N.; Kim, T.; Afshari, N.A. Microbiological profiles of fungal keratitis: A 10-year study at a tertiary referral center. J. Ophthalmic. Inflamm. Infect. 2016, 6, 5. [CrossRef] [PubMed]

49. Satpathy, G.; Ahmed, N.H.; Nayak, N.; Tandon, R.; Sharma, N.; Agarwal, T.; Vanathi, M.; Titiyal, J.S. Spectrum of mycotic keratitis in north India: Sixteen years study from a tertiary care ophthalmic centre. J. Infect. Public Health 2019, 12, 367-371. [CrossRef]

50. Rai, M.; Ingle, A.P.; Ingle, P.; Gupta, I.; Mobin, M.; Bonifaz, A.; Alves, M. Recent advances on mycotic keratitis caused by dematiaceous hyphomycetes. J. Appl. Microbiol. 2021. [CrossRef]

51. Nath, R.; Baruah, S.; Saikia, L.; Devi, B.; Borthakur, A.K.; Mahanta, J. Mycotic corneal ulcers in upper Assam. Indian J. Ophthalmol 2011, 59, 367-371. [CrossRef] [PubMed]

52. Yuan, X.; Wilhelmus, K.R.; Matoba, A.Y.; Alexandrakis, G.; Miller, D.; Huang, A.J. Pathogenesis and outcome of Paecilomyces keratitis. Am. J. Ophthalmol. 2009, 147, 691-696.e3. [CrossRef]

53. Chew, R.; Dorman, A.; Woods, M.L. Purpureocillium lilacinum keratitis: A case series and review of the literature. Can. J. Ophthalmol. 2016, 51, 382-385. [CrossRef] [PubMed]

54. Roth, M.; Holtmann, C.; Daas, L.; Kakkassery, V.; Kurzai, O.; Geerling, G. Results from the German Fungal Keratitis Registry: Significant differences between cases with and without history of contact lens use. Cornea 2021. Publish Ahead of Print. [CrossRef]

55. Gajjar, D.U.; Pal, A.K.; Ghodadra, B.K.; Vasavada, A.R. Microscopic evaluation, molecular identification, antifungal susceptibility, and clinical outcomes in Fusarium, Aspergillus and dematiaceous keratitis. Biomed. Res. Int. 2013, 2013, 605308. [CrossRef]

56. Hassan, A.S.; Al-Hatmi, A.M.; Shobana, C.S.; van Diepeningen, A.D.; Kredics, L.; Vagvolgyi, C.; Homa, M.; Meis, J.F.; de Hoog, G.S.; Narendran, V.; et al. Antifungal susceptibility and phylogeny of opportunistic members of the genus Fusarium causing human keratomycosis in South India. Med. Mycol. 2016, 54, 287-294. [CrossRef] [PubMed]

57. Herkert, P.F.; Al-Hatmi, A.M.S.; de Oliveira Salvador, G.L.; Muro, M.D.; Pinheiro, R.L.; Nucci, M.; Queiroz-Telles, F.; de Hoog, G.S.; Meis, J.F. Molecular characterization and antifungal susceptibility of clinical Fusarium species from Brazil. Front. Microbiol. 2019, 10, 737. [CrossRef]

58. Chen, Y.T.; Yeh, L.K.; Ma, D.H.K.; Lin, H.C.; Sun, C.C.; Tan, H.Y.; Chen, H.C.; Chen, S.Y.; Sun, P.L.; Hsiao, C.H. Paecilomyces/Purpureocillium keratitis: A consecutive study with a case series and literature review. Med. Mycol. 2020, 58, 293-299. [CrossRef]

59. Cuenca-Estrella, M.; Gomez-Lopez, A.; Mellado, E.; Buitrago, M.J.; Monzon, A.; Rodriguez-Tudela, J.L. Scopulariopsis brevicaulis, a fungal pathogen resistant to broad-spectrum antifungal agents. Antimicrob. Agents Chemother. 2003, 47, 2339-2341. [CrossRef]

60. Lalitha, P.; Shapiro, B.L.; Srinivasan, M.; Prajna, N.V.; Acharya, N.R.; Fothergill, A.W.; Ruiz, J.; Chidambaram, J.D.; Maxey, K.J.; Hong, K.C.; et al. Antimicrobial susceptibility of Fusarium, Aspergillus, and other filamentous fungi isolated from keratitis. Arch. Ophthalmol. 2007, 125, 789-793. [CrossRef]

61. Lalitha, P.; Vijaykumar, R.; Prajna, N.V.; Fothergill, A.W. In vitro natamycin susceptibility of ocular isolates of Fusarium and Aspergillus species: Comparison of commercially formulated natamycin eye drops to pharmaceutical-grade powder. J. Clin. Microbiol. 2008, 46, 3477-3478. [CrossRef]

62. Oliveira Dos Santos, C.; Kolwijck, E.; van der Lee, H.A.; Tehupeiory-Kooreman, M.C.; Al-Hatmi, A.M.S.; Matayan, E.; Burton, M.J.; Eggink, C.A.; Verweij, P.E. In vitro activity of chlorhexidine compared with seven antifungal agents against 98 Fusarium isolates recovered from fungal keratitis patients. Antimicrob. Agents Chemother. 2019, 63. [CrossRef]

63. Te Welscher, Y.M.; Jones, L.; van Leeuwen, M.R.; Dijksterhuis, J.; de Kruijff, B.; Eitzen, G.; Breukink, E. Natamycin inhibits vacuole fusion at the priming phase via a specific interaction with ergosterol. Antimicrob. Agents Chemother. 2010, 54, $2618-2625$. [CrossRef] [PubMed] 
64. Te Welscher, Y.M.; ten Napel, H.H.; Balague, M.M.; Souza, C.M.; Riezman, H.; de Kruijff, B.; Breukink, E. Natamycin blocks fungal growth by binding specifically to ergosterol without permeabilizing the membrane. J. Biol. Chem. 2008, 283, 6393-6401. [CrossRef] [PubMed]

65. Alastruey-Izquierdo, A.; Cuenca-Estrella, M.; Monzon, A.; Mellado, E.; Rodriguez-Tudela, J.L. Antifungal susceptibility profile of clinical Fusarium spp. isolates identified by molecular methods. J. Antimicrob. Chemother. 2008, 61, 805-809. [CrossRef] [PubMed]

66. Ni, N.; Nam, E.M.; Hammersmith, K.M.; Nagra, P.K.; Azari, A.A.; Leiby, B.E.; Dai, Y.; Cabrera, F.A.; Ma, J.F.; Lambert, C.E., Jr.; et al. Seasonal, geographic, and antimicrobial resistance patterns in microbial keratitis: 4-year experience in eastern Pennsylvania. Cornea 2015, 34, 296-302. [CrossRef] [PubMed]

67. Tortorano, A.M.; Prigitano, A.; Dho, G.; Esposto, M.C.; Gianni, C.; Grancini, A.; Ossi, C.; Viviani, M.A. Species distribution and in vitro antifungal susceptibility patterns of 75 clinical isolates of Fusarium spp. from northern Italy. Antimicrob. Agents Chemother. 2008, 52, 2683-2685. [CrossRef] [PubMed] 